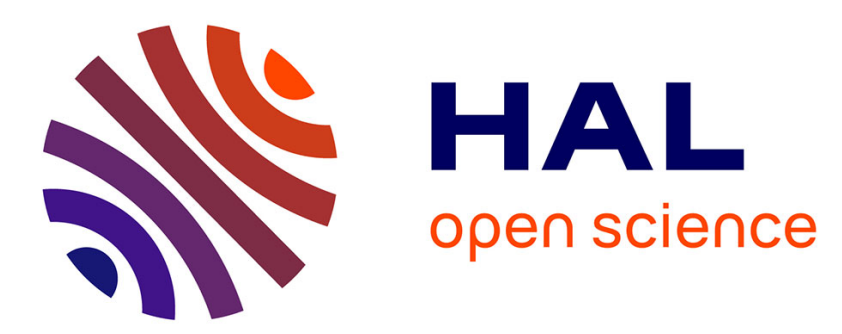

\title{
Quelle est la spécificité des discours électoraux? Le cas de Stephen Harper
}

Dominique Labbé, Denis Monière

\section{To cite this version:}

Dominique Labbé, Denis Monière. Quelle est la spécificité des discours électoraux? Le cas de Stephen Harper. Canadian Journal of Political Science / Revue canadienne de science politique, 2010, 43 (1), pp.69-86. halshs-00470154

\section{HAL Id: halshs-00470154 \\ https://shs.hal.science/halshs-00470154}

Submitted on 4 Apr 2010

HAL is a multi-disciplinary open access archive for the deposit and dissemination of scientific research documents, whether they are published or not. The documents may come from teaching and research institutions in France or abroad, or from public or private research centers.
L'archive ouverte pluridisciplinaire HAL, est destinée au dépôt et à la diffusion de documents scientifiques de niveau recherche, publiés ou non, émanant des établissements d'enseignement et de recherche français ou étrangers, des laboratoires publics ou privés. 
Dominique Labbé

(Institut d'études politiques de Grenoble)

Denis Monière

(Université de Montréal)

\title{
Quelle est la spécificité des discours électoraux? Le cas de Stephen Harper
}

\author{
Canadian Journal of Political Science / Revue canadienne de science \\ politique, 43:1, (March/ mars 2010), p. 69-86.
}

Résumé : Cette étude de cas démontre que le discours électoral possède des caractéristiques propres. On donne l'exemple de Stephen Harper dont les discours tenus lors des élections de 2008 se différencient de ceux qu'il a prononcés à titre de chef de gouvernement. Le discours électoral est plus ancré socialement. C'est aussi un discours qui valorise le collectif national : le locuteur privilégie l'emploi du «nous», plutôt que de se présenter comme principal responsable des choix collectifs. Comparativement aux discours gouvernementaux, le discours électoral est aussi moins abstrait et plus orienté vers l'action, comme l'indique la prédominance du groupe verbal sur le groupe nominal. La forte présence de la construction négative et la désignation des adversaires (noms propres) soulignent le caractère polémique du discours électoral.

Abstract: This case study demonstrates that electoral speeches possess specific characteristics. We give the example of Stephen Harper's speeches, given during the 2008 elections, which differ from those delivered when he was prime minister. The electoral speech is more socially anchored. It values the nation. When he is campaigning, S. Harper also uses the pronoun "we" more frequently, so that he does not appear as the main decider of collective choices. Compared with governmental speeches, electoral speeches are also less theoretical and more action orientated as indicated by the predominance of verbal over nominal groups. The very frequent presence of the negative construction and the use of opponents' names highlight the polemic character of electoral speeches.

Mots clefs : discours politique ; discours electoral ; lexicometrie ; Canada ; Harper Political discourses ; electoral speeches ; lexical statistics ;

Version de septembre 2009

Telle qu'elle a été soumise à la rédaction de la revue

Pour toute citation se reporter à la revue papier qui fait foi 
Le discours est un ingrédient indispensable de la vie politique. C'est l'instrument privilégié des acteurs politiques tant pour conquérir le pouvoir que pour l'exercer. Les partis et les candidats s'en servent pour définir leurs offres de politiques, consolider leur base électorale et attirer de nouveaux électeurs. Une fois élus, ils gouvernent aussi par le discours en expliquant leurs choix aux citoyens.

Le style discursif déployé en campagne électorale pour convaincre diffère-t-il de celui qui est employé pour gouverner? Et en quoi? La réponse à ces deux questions n'est pas évidente, car il y a fort peu d'études portant sur les propos tenus en campagne électorale et jusqu'à présent ces recherches visaient à déterminer le degré de fiabilité du discours électoral : il s'agissait de savoir dans quelle mesure ce qui avait été promis en campagne électorale avait été ensuite réalisé par le parti élu (Monière, 1988).

Pour comparer les discours électoraux et les discours gouvernementaux, il faut neutraliser les effets du temps, des locuteurs et des idéologies. Cela implique que la comparaison s'effectue sur des messages émis par les mêmes personnes et dans une séquence temporelle restreinte qui inclut une période électorale et les mois qui suivent ou qui précèdent l'élection.

L'étude des discours électoraux a été négligée jusqu'à présent, car on ne disposait pas de corpus suffisamment étendus : l'accès à ce type de textes était difficile; le chercheur ne pouvait pas suivre toutes les équipes de campagne afin de recueillir les messages émis par les candidats; l'enregistrement et la transcription des verbatim étaient onéreux. Aujourd'hui, il est devenu possible de constituer des «corpus» (vastes ensembles de textes transcrits intégralement suivant des procédures standardisées). En effet, les campagnes se déroulent aussi sur Internet, média que les partis et les chefs utilisent pour diffuser les interventions qu'ils jugent importantes. Ainsi, les textes diffusés sur Internet ont-ils servi à plusieurs études sur la campagne présidentielle française de 2007 (Labbé et Monière, 2008; Coullomb-Gully, 2009).

Il devient donc possible de rassembler chronologiquement les discours électoraux et de les comparer à ceux qui ont été ou qui seront prononcés par les mêmes personnes lorsqu'elles seront à la tête de l'exécutif. C'est une étude de ce genre qui a été réalisée sur le premier ministre canadien, Stephen Harper. Une autre est en cours sur Nicolas 
Sarkozy, actuel président de la République française (les premiers résultats de cette étude fourniront un étalon de comparaison avec S. Harper).

Dans quelle mesure la dynamique électorale induit-elle des comportements discursifs et des stratégies spécifiques de communication? Le vocabulaire utilisé en campagne électorale est-il plus redondant ou plus stéréotypé que celui qui est employé par les politiciens en temps ordinaire? Quelles sont les particularités du langage électoral? La statistique appliquée au langage permet d'apporter des réponses à ces questions. L'intérêt de cette démarche réside dans son caractère formalisé, systématique et reproductible. Nous tenterons d'en montrer l'intérêt, pour la science politique, grâce à une comparaison des vocabulaires utilisés par S. Harper comme premier ministre, puis comme chef de parti en campagne électorale. Cette analyse sera complétée par des portraits grammaticaux et stylistiques du gouvernant, puis du chef en campagne.

\section{Corpus et traitement}

Le corpus se compose des 52 discours prononcés par S. Harper entre janvier et octobre 2008, répartis en deux sous-corpus. D'une part, 29 interventions comme premier ministre avant le 7 septembre 2008 (annonce des élections législatives), soit 36266 mots, composent le sous-corpus des discours de gouvernement. D'autre part, les 23 discours prononcés durant la campagne (du 7 septembre au 14 octobre 2008), forment le sous-corpus des discours de campagne (soit 25128 mots). Les discours du premier ministre étant diffusés en français et en anglais, nous avons utilisé la version française. Les textes sont en ligne sur le site de la Société du patrimoine politique du Québec (www.archivespolitiquesduquebec.com).

Tous ces textes ont subi le même traitement (D. Labbé, 1990a). Après une correction orthographique, chaque texte a été doté d'un en-tête indiquant l'identité du locuteur, la date du discours et le lieu où il a été prononcé. Enfin, chaque mot du texte a été lemmatisé. La lemmatisation attache à chacun de ces mots une étiquette comportant la forme canonique - l'entrée sous laquelle il peut être trouvé dans un dictionnaire de langue - et la catégorie grammaticale qui accompagne généralement cette entrée de dictionnaire (D. Labbé, 1990b). Par exemple, toutes les flexions d'un même verbe sont rassemblées sous l'infinitif de ce verbe.

L'étiquetage des mots est réalisé par un programme d'analyse morphosyntaxique des éléments de la phrase. Cette lemmatisation n'a pas comme seul intérêt de lever les 
ambiguïtés. En standardisant les individus à étudier (les vocables) et en réduisant leur nombre, la lemmatisation facilite l'analyse statistique. Elle permet ainsi d'accéder au vocabulaire d'un homme politique comme S. Harper et, ici, d'analyser les changements survenus lors du passage du statut de premier ministre à celui de chef de parti en campagne électorale.

\section{Les vocabulaires de gouvernement et de campagne chez Stephen Harper}

Les vocables les plus fréquents offrent un premier aperçu. Les cinq tableaux cidessous permettent de comparer les vocables les plus utilisés par S. Harper comme premier ministre, puis comme candidat. Les deux sous-corpus n'ayant pas la même longueur, il est nécessaire de convertir le nombre absolu d'occurrences des vocables en fréquences relatives (ici en proportion pour mille mots : \%o). Par exemple, cette conversion permet de constater que le premier ministre utilisait plus le mot Canada (tableau 1, première ligne, cadre de gauche : 5,6 \%o) que ne l'a fait le candidat $(4,7 \%$ ) et qu'à l'inverse, Canadien(s, ne, nes) a été privilégié pendant la campagne électorale. De même, on voit que le thème États-Unis a été au cœur de ce discours électoral - en troisième position dans la partie droite du tableau - alors qu'il est absent de la partie gauche, car trop peu cité dans les discours de gouvernement. En fait, le chef du Parti conservateur promettait de protéger les Canadiens de la crise venue des États-Unis. Comme on le verra plus bas, il s'agissait de son principal thème de campagne. 


\section{TABLEAU 1}

\section{LES NOMS PROPRES LES PLUS UTILISÉS}

\begin{tabular}{|c|c|c|c|c|c|}
\hline Premier ministre & Fréquence & $\%$ & & Candidat Fréquence & $\%$ \\
\hline Canada & 202 & 5,6 & Canada & 119 & 4,7 \\
\hline Canadien & 104 & 2,9 & Canadien & 114 & 4,5 \\
\hline Arctique & 30 & 0,8 & États-Unis & 20 & 0,8 \\
\hline Ottawa & 17 & 0,5 & TPS & 9 & 0,4 \\
\hline Fiducie & 16 & 0,4 & Saint John & 7 & 0,3 \\
\hline Colombie-Britannique & 14 & 0,4 & Dion & 6 & 0,2 \\
\hline Montréal & 14 & 0,4 & Fredericton & 6 & 0,2 \\
\hline Creighton & 13 & 0,4 & Arctique & 5 & 0,2 \\
\hline Diefenbaker & 13 & 0,4 & Bloc & 5 & 0,2 \\
\hline Pologne & 13 & 0,4 & G7 & 5 & 0,2 \\
\hline
\end{tabular}

Dans tout corpus en français, les verbes être, avoir et faire sont les plus utilisés et figurent toujours dans cet ordre dans les listes des vocables classés par fréquence. Ainsi, la manière dont $\mathrm{S}$. Harper a utilisé le verbe aller durant sa campagne électorale paraîtelle tout à fait singulière (tableau 2). Dans le vocabulaire du premier ministre, ce verbe figurait à la $18^{\mathrm{e}}$ position, avec une fréquence de $0,9 \%$ (de ce fait, il est absent du tableau 2, qui est limité aux dix verbes les plus fréquents); sa fréquence a été multipliée par sept dans le vocabulaire de la campagne électorale, ce qui l'a fait passer à la troisième position, avant faire.

Le verbe aller permet de situer l'action dans le futur en donnant à la phrase un tour familier. Par exemple, chez S. Harper : nous n'allons pas augmenter les taxes (au lieu de «nous n'augmenterons pas les taxes»). La très forte fréquence de cette tournure montre donc que la campagne de S. Harper était orientée vers le futur (plus que sur le bilan de son action passée).

On note également l'occurrence, dans la liste, de verbes caractéristiques du discours électoral en temps de crise : protéger, augmenter, aider, offrir. 
TABLEAU 2

\section{LES VERBES LES PLUS UTILISÉS}

\begin{tabular}{|c|c|c|c|c|c|c|}
\hline Premier ministre & Fréquence & $\%$ & & Candidat & Fréquence & $\%$ \\
\hline être & 705 & 19,4 & être & & 447 & 17,8 \\
\hline avoir & 655 & 18,1 & avoir & & 424 & 16,9 \\
\hline faire & 136 & 3,8 & aller & & 153 & 6,1 \\
\hline pouvoir & 92 & 2,5 & faire & & 145 & 5,8 \\
\hline permettre & 64 & 1,8 & pouvoir & & 71 & 2,8 \\
\hline dire & 58 & 1,6 & devoir & & 70 & 2,8 \\
\hline devoir & 57 & 1,6 & protéger & & 63 & 2,5 \\
\hline remercier & 47 & 1,3 & augmenter & & 56 & 2,2 \\
\hline prendre & 40 & 1,1 & aider & & 51 & 2,0 \\
\hline mettre & 39 & 1,1 & offrir & & 48 & 1,9 \\
\hline
\end{tabular}

Les substantifs les plus fréquents (tableau 3) résument les principaux thèmes abordés dans le discours. Aussi enregistrent-ils bien les changements induits par l'entrée en campagne électorale. Les deux listes n'ont que trois vocables en commun : gouvernement, Canada et Canadien (s, ne, nes). Le plan - quasiment absent du discours de gouvernement - arrive en première place dans le discours électoral. En effet, le Parti conservateur qui avait lancé la campagne électorale dans un climat de relative confiance économique fut obligé de rajuster son discours, à la suite de la chute des bourses mondiales qui annonçait de fortes perturbations économiques. Le gouvernement a donc improvisé un plan pour protéger l'économie de la crise. En fait, ce plan renforçait les mesures déjà annoncées, notamment en faveur des familles, et réitérait la promesse de ne pas augmenter les taxes.

Ces principaux thèmes sont confirmés par la liste des adjectifs les plus utilisés (tableau 4). On notera également la place prépondérante, dans le discours de S. Harper, de l'adjectif nouveau, qui était beaucoup moins fréquent dans la plupart des vocabulaires autres que celui de la politique (sauf celui de la publicité). 
TABLEAU 3

\section{LES SUBSTANTIFS LES PLUS UTILISÉS}

\begin{tabular}{|lcc|lccc|}
\hline \multicolumn{1}{|c|}{ Premier ministre } & Fréquence & $\% 0$ & & Candidat & Fréquence & $\% 0$ \\
\hline Canada & 202 & 5,6 & plan & 159 & 6,3 \\
gouvernement & 130 & 3,6 & gouvernement & 141 & 5,6 \\
pays & 110 & 3,0 & Canada & 119 & 4,7 \\
Canadien & 104 & 2,9 & Canadien & 114 & 4,5 \\
merci & 80 & 2,2 & économie & 93 & 3,7 \\
monsieur & 79 & 2,2 & famille & 91 & 3,6 \\
ministre & 73 & 2,0 & mesure & 76 & 3,0 \\
économie & 71 & 2,0 & dollar & 73 & 2,9 \\
budget & 69 & 1,9 & pays & 73 & 2,9 \\
année & 65 & 1,8 & taxe & 56 & 2,2 \\
\hline
\end{tabular}

TABLEAU 4

LES ADJECTIFS LES PLUS UTILISÉS

\begin{tabular}{|c|c|c|c|c|c|c|}
\hline Premier ministre & Fréquence & $\%$ & & Candidat & Fréquence & $\%$ \\
\hline canadien & 102 & 2,8 & nouveau & & 102 & 4,1 \\
\hline nouveau & 94 & 2,6 & canadien & & 87 & 3,5 \\
\hline grand & 87 & 2,4 & économique & & 76 & 3,0 \\
\hline économique & 60 & 1,7 & conservateur & & 70 & 2,8 \\
\hline national & 51 & 1,4 & fiscal & & 70 & 2,8 \\
\hline bon & 42 & 1,2 & mondial & & 54 & 2,1 \\
\hline dernier & 40 & 1,1 & dernier & & 33 & 1,3 \\
\hline important & 35 & 1,0 & grand & & 32 & 1,3 \\
\hline fiscal & 34 & 0,9 & financier & & 31 & 1,2 \\
\hline meilleur & 34 & 0,9 & national & & 27 & 1,1 \\
\hline
\end{tabular}

Le tableau 5 met en valeur une caractéristique propre au discours de la majorité des premiers ministres canadiens, comme québécois : l'insistance sur le nous et le relatif effacement de la première personne du singulier (je, me, moi). À l'inverse, les premiers ministres et les présidents français privilégient cette première personne du singulier (D. Labbé, 1990a : 32-36; Mayaffre, 2004 : 76-85). Cela est également vrai de la part des candidats à l'élection présidentielle française de 2007 (Labbé et Monière, 2008b). En 
moyenne, les dirigeants politiques français utilisent le je deux fois plus que leurs homologues de la fédération canadienne ou du Québec. Cette différence considérable pourrait s'expliquer par les différences entre la collégialité, au moins de façade, imposée par le système parlementaire et la personnalisation inhérente au système présidentiel. Certes, dans le système de Westminster, le premier ministre concentre beaucoup de pouvoirs entre ses mains, mais c'est en tant que chef d'une majorité parlementaire qu'il se doit d'incarner au moins en paroles.

\section{TABLEAU 5}

\section{LES PRONOMS LES PLUS UTILISÉS}

\begin{tabular}{|lcc|lcc|}
\hline Premier ministre & Fréquence & $\%$ Candidat & Fréquence & $\% 0$ \\
\hline nous & 459 & 12,7 & nous & 440 & 17,5 \\
je & 342 & 9,4 & qui & 228 & 9,1 \\
qui & 297 & 8,2 & je & 139 & 5,5 \\
ce & 221 & 6,1 & ce & 138 & 5,5 \\
que & 207 & 5,7 & il & 117 & 4,7 \\
il & 178 & 4,9 & que & 116 & 4,6 \\
vous & 148 & 4,1 & vous & 74 & 2,9 \\
se & 145 & 4,0 & se & 71 & 2,8 \\
le & 130 & 3,6 & le & 63 & 2,5 \\
ils & 123 & 3,4 & ils & 58 & 2,3 \\
\hline
\end{tabular}

Tout locuteur peut entretenir avec le destinataire de son discours deux types de relations (Benveniste, 1956; Kerbat-Orecchioni, 1988 : 40-44; Benoît et Al, 1985). D'une part, la tension interlocutive (je-vous) sépare l'émetteur du destinataire et souligne le sens de l'interlocution (le je est la source, le vous est la cible). D'autre part, la tension inclusive (nous) fond l'émetteur dans un groupe plus large : le gouvernement, la majorité parlementaire, le parti, souvent l'ensemble des Canadiens et Canadiennes. La personne du locuteur s'efface derrière une entité plus large qui, dans le cas des premiers ministres, est la communauté nationale à laquelle ils prêtent leur voix. C'est donc ce second type de relations que S. Harper a privilégié lors de sa campagne électorale, plus encore que dans ses discours gouvernementaux. 
Chez le candidat $\mathrm{S}$. Harper, le mécanisme d'inclusion fonctionne d'après le schéma suivant : «je demande aux Canadiens de choisir le parti qui a un plan pour notre avenir» (10 octobre 2008). La même construction - avec de légères variantes - est utilisée pendant toute la campagne. Le locuteur assume son statut de source du discours, en ouvrant la phrase par un je, mais il se fond parmi les Canadiens et poursuit avec le nous dénotant la communauté nationale dont il serait le porte-parole.

À l'issue de cette synthèse, une question demeure : les écarts de fréquence constatés entre les discours gouvernementaux et les discours électoraux peuvent-ils provenir de fluctuations normales comme on en rencontre dans tout phénomène naturel? Ou bien ces écarts dépassent-ils les marges de fluctuation normales, signalant ainsi un changement de stratégie de communication? Dans ce cas, quels sont les vocables les plus caractéristiques de l'un ou l'autre de ces deux types de discours? Un calcul statistique simple permet de répondre à ces questions évidentes, mais jamais abordées en «analyse du discours».

\section{Le vocabulaire caractéristique de la campagne électorale}

Le raisonnement est le suivant. Quand il était premier ministre, S. Harper a utilisé 342 pronoms de la première personne du singulier (je) pour 36266 mots au total (soit en moyenne 9,4 fois pour 1000 mots). S'il avait gardé le même rythme d'utilisation dans ses discours de campagne (soit 25168 mots), ceux-ci auraient dû contenir :

$$
342 * \frac{25168}{36266}=237 \text { pronoms je }
$$

Or, pendant cette campagne, S. Harper n'a employé que 139 je, soit 98 de moins que ce qui était attendu en fonction de sa performance comme premier ministre. Un test statistique (Labbé et Labbé, 1994) indique qu'un tel écart a moins d'une chance sur 10000 d'être le fruit du hasard. On peut donc en conclure, avec une chance négligeable de se tromper, que $\mathrm{S}$. Harper a réduit significativement l'emploi de la première personne dans ses discours électoraux. À l'inverse, l'augmentation du pronom nous est tout aussi significative du point de vue statistique. Ce qui suggère que $S$. Harper a plus tendance à s'affirmer personnellement lorsqu'il est chef du gouvernement et à se fondre dans un collectif - ici les Canadiens - lorsqu'il est en campagne électorale. Le chef en campagne électorale joue donc la carte de la modestie apparente. En fait, s'il se fond, en paroles, 
dans un tout qui englobe l'auditoire, c'est pour mieux convaincre les électeurs de s'identifier à lui.

De manière générale, si un vocable est notablement sur-employé, on en déduit que la stratégie électorale du leader conservateur a privilégié ce mot et les notions qui lui sont associées. À l'inverse, s'il est notablement sous-employé, c'est qu'il appartient au vocabulaire de gouvernement et que S. Harper a préféré l'éviter lors de la campagne électorale.

Ce calcul confirme les conclusions suggérées par les mots les plus fréquents. En campagne électorale, le premier ministre a privilégié les Canadiens et moins parlé du Canada; les verbes aller, faire et devoir sont passés au premier plan; S. Harper a préféré l'inclusion (nous, notre) à l'interlocution (je-vous); il a développé un discours centré sur les questions économiques et fiscales. Parmi les caractéristiques du discours électoral, on note également l'augmentation considérable de la construction «ne... pas» et «ne... plus» (Ducrot, 1972 : 216-217; Achard et Al, 1995). En utilisant une négation, on rejette - implicitement ou explicitement - un énoncé que l'on prête à d'autres, énoncé dont on dénonce la fausseté ou la nocivité. L'abondance de ces constructions négatives signifie donc que, durant la campagne électorale, S. Harper a consacré une part importante de ses discours à rejeter les propositions des autres partis (essentiellement les Libéraux, accusés de vouloir augmenter les impôts et creuser les déficits). Même s'il s'en est défendu à plusieurs reprises, le chef conservateur, comme ceux de tous les autres partis, avait donc choisi de centrer sa campagne sur la critique des autres, plus que sur des propositions positives (Monière, 2008). Cette logique polémique, caractéristique du discours électoral, est aussi attestée par un autre indicateur. Dans le tableau 1 ci-dessus (noms propres les plus fréquents), on retrouve les noms de ses adversaires (Dion et le Bloc Québécois).

Des phrases caractéristiques peuvent donner de la chair à ces listes, à la manière dont les dictionnaires illustrent le sens des mots par des citations des bons auteurs. Naturellement, ces phrases ne doivent pas être choisies arbitrairement par l'observateur (comme elles le sont dans les exempliers utilisés en analyse du discours). On doit avoir la certitude qu'elles sont bien les plus caractéristiques du corpus étudié. Pour cela, le logiciel relit tout le sous-corpus en affectant un score à chaque phrase en fonction du nombre de vocables caractéristiques qu'elle contient. Ce score augmente de 1 à la 
rencontre d'un vocable significativement sur-employé et diminue de 1 si le vocable est significativement sous-employé. Les phrases qui ont les scores les plus élevés sont donc les plus caractéristiques du sous-corpus (ici les discours électoraux). Voici la phrase dont le score est le plus haut :

«Le 14 octobre, je demande aux Canadiens de choisir le parti qui a un plan pour notre avenir, le parti qui protégera les familles canadiennes, le parti qui saura gérer notre économie pendant une crise mondiale» (10 octobre 2008).

Sur les 36 mots composant cette phrase, 19 appartiennent au vocabulaire caractéristique du discours électoral : Canadien(s, ne, nes), l'adjectif canadien, les verbes choisir, protéger, gérer, les substantifs parti, plan, économie, famille, crise, mondial, le possessif notre... En revanche, un vocable est significativement sousemployé, le je (mais sa présence se limite à l'énonciation initiale selon le procédé déjà mis en lumière). Le score absolu de cette phrase s'établit donc à 18 et le score relatif à 50 pour cent (18/36). Le score absolu avantage les phrases les plus longues. Mais comme ce sont aussi celles qui sont les plus pensées, ces phrases caractéristiques révèlent les thèmes qui occupent l'esprit du locuteur et la manière dont il $a$, consciemment, décidé de les mettre en valeur devant son auditoire (Labbé et Monière, 2007). La répétition du même thème dans plusieurs phrases longues de S. Harper apporte d'ailleurs une confirmation, comme le montrent ces deux phrases également isolées par le logiciel :

«Les électeurs vont choisir entre le plan économique des Conservateurs, qui offre des avantages modestes, réalistes, abordables et crédibles aux familles canadiennes, et des stratagèmes risqués et coûteux financés par des déficits ou de nouvelles taxes, notamment une nouvelle taxe sur le carbone» (24 septembre 2008).

«Chers amis, le 14 octobre, en une période d'incertitude économique mondiale, je vous demande de choisir le seul parti qui a un plan prudent, qui a fait ses preuves, un leader, un parti qui comprennent notre économie, qui vont la protéger et qui vont défendre les intérêts des familles canadiennes» (11 octobre 2008).

Enfin, il existe une autre manière de comparer les discours gouvernementaux et électoraux : les catégories grammaticales. 


\section{Densité des catégories grammaticales}

Rappelons qu'avant d'entrer dans une base de données lexicales, chaque mot du texte reçoit une étiquette comportant notamment sa catégorie grammaticale. Cette information permet de dresser une sorte de carte d'identité des corpus et des locuteurs (tableau 6).

TABLEAU 6

STEPHEN HARPER PREMIER MINISTRE ET CANDIDAT. COMPARAISON DES DENSITÉS RELATIVES D'EMPLOI DES CATÉGORIES GRAMMATICALES (\%)

\begin{tabular}{|l|c|c|c|}
\hline Catégories & $\begin{array}{c}\text { A } \\
\text { Premier m. }(\%)\end{array}$ & $\begin{array}{c}\text { B } \\
\text { Candidat }(\%)\end{array}$ & $\begin{array}{c}\text { C } \\
\text { (B-A)/A (\%) }\end{array}$ \\
\hline Verbes & 124,3 & 136,5 & $+9,8$ \\
Formes fléchies & 71,0 & 75,7 & $+6,7$ \\
Participes passés & 21,1 & 15,4 & $-27,1$ \\
Participes présents & 3,4 & 3,9 & $+15,2$ \\
Infinitifs & 28,7 & 41,4 & $+44,0$ \\
\hline Noms propres & 34,9 & 20,0 & $-42,6$ \\
\hline Noms communs & 207,9 & 219,0 & $+5,3$ \\
\hline Adjectifs & 74,0 & 84,1 & $+13,6$ \\
Adjectifs participes passés & 8,9 & 9,6 & $+7,7$ \\
\hline Pronoms & 77,4 & 68,8 & $-11,2$ \\
Pronoms personnels & 40,8 & 37,8 & $-7,5$ \\
Pronoms démonstratifs & 8,8 & 7,6 & $-13,1$ \\
Pronoms possessifs & 0,1 & 0,1 & $-42,3$ \\
Pronoms indéfinis & 2,9 & 1,8 & $-38,7$ \\
Pronoms relatifs & 19,5 & 17,8 & $-8,8$ \\
\hline Déterminants & 195,0 & 205,6 & $+5,4$ \\
Articles & 140,4 & 147,6 & $+5,1$ \\
Nombres & 18,7 & 21,8 & $+16,4$ \\
Possessifs & 19,5 & 21,0 & $+7,6$ \\
Démonstratifs & 9,4 & 8,7 & $-7,2$ \\
Indéfinis & 7,0 & 6,5 & $-6,8$ \\
\hline Adverbes & 51,3 & 44,5 & $-13,4$ \\
\hline Prépositions & 179,8 & 169,9 & $-5,5$ \\
\hline Conjonctions & 54,5 & 51,6 & $-5,3$ \\
Coordination & 40,2 & 36,5 & $-9,0$ \\
Subordination & 14,4 & 15,1 & $+5,0$ \\
\hline Mots étrangers & 0,8 & 0,1 & $-84,5$ \\
\hline
\end{tabular}


Le raisonnement est le suivant. Avant septembre 2008, lorsque S. Harper était premier ministre, il employait en moyenne 124,3 verbes pour 1.000 mots (colonne A). Dans ses discours comme candidat, cette moyenne est de 136,5 (colonne B), soit une augmentation d'environ un dixième (colonne $\mathrm{C}$ ). Cette progression est statistiquement significative avec une marge d'erreur inférieure à 1 pour cent.

Cette progression du verbe est la résultante de mouvements contrastés décrits dans les quatre lignes suivantes. Le présent et le futur (formes fléchies) progressent de près de 7 pour cent alors que le passé recule de plus d'un quart (- $27 \%)$. Autrement dit, S. Harper a fait campagne non pas sur le passé (son bilan), mais sur la situation (la crise) et sur les enjeux pour le futur (son plan comparé aux programmes des autres partis). L'augmentation la plus importante affecte surtout l'infinitif. Ce mode se rencontre dans deux types de phrase : le slogan et la modalisation.

Le slogan consiste à supprimer le sujet et à placer le verbe en début de phrase. Par exemple : «Protéger l'économie canadienne contre la crise financière aux États-Unis» (répété trois fois : le 3 octobre 2008, le 10 octobre 2008 et le 11 octobre 2008). Une autre construction obtient un effet presque équivalent, soit «préposition + infinitif». Par exemple : «Notre gouvernement exécute un plan pour garder une économie solide» (8 octobre 2008). Chaque préposition donne une orientation à la phrase. La préposition pour - qui est le sur-emploi le plus caractéristique du discours électoral - est, en français, la manière la plus économique d'indiquer un geste en faveur de.

Deuxièmement, la modalisation associe un verbe fléchi à un infinitif (Benveniste, 1965). Par exemple : «Nous devons garder un faible fardeau fiscal» (10 octobre 2008). «Nous, on veut maintenir un faible fardeau fiscal et renforcer l'économie canadienne» (9 octobre 2008). La teinte est donnée par le premier verbe qualifié de «pseudo auxiliaire». Ces verbes sont très nombreux, mais peuvent être répartis en quatre groupe principaux suivant qu'ils expriment la nécessité (falloir), la volonté (vouloir), l'obligation (devoir) et la connaissance (savoir). Les deux modalités les plus significatives du discours électoral de S. Harper sont l'obligation (devoir) et la volonté (vouloir). Comme elles sont sous-employées dans le discours gouvernemental, il s'agit d'une thématique électorale et non d'une caractéristique propre au premier ministre. Autrement dit, c'est lorsqu'il est en campagne électorale que le chef politique exprime sa volonté d'agir tout en se conformant aux obligations et aux engagements qu'il prend... 
Le tableau 6 révèle également que S. Harper utilise beaucoup moins de noms propres (vocables commençant par une majuscule initiale) en période électorale (-43\%) que dans ses discours de premier ministre). Les noms propres n'appartiennent pas à proprement parler à la langue, mais à la réalité extérieure au discours. Les toponymes assurent l'ancrage du discours dans l'espace; les patronymes l'ancrent dans la société; les sigles et les noms commerciaux, dans l'économie, et ainsi de suite Autrement dit, le leader conservateur a choisi un discours de campagne peu situé dans l'espace et dans la société, c'est-à-dire une certaine généralité, voire une certaine abstraction.

En revanche, l'espace économique est bien présent sous une autre forme, soit les nombres $(+16,4 \%)$, que l'on peut relier au sur-emploi de substantifs comme budget ou dollar. Cela confirme que S. Harper a centré sa campagne sur la situation économique.

Il est possible de synthétiser ces mouvements grâce à un indicateur simple : le partage de la surface du texte entre le verbe et le nom.

\section{Nom ou verbe?}

Les groupes nominal et verbal sont opposés. Le premier groupe est constitué des pronoms, des verbes, des adverbes et des conjonctions de subordination; le second comporte les substantifs, adjectifs, déterminants, prépositions et conjonctions de coordination. Certes, le partage n'est pas exclusif : certains adverbes peuvent se glisser dans le groupe nominal, certaines prépositions dans le groupe verbal, certains pronoms relatifs s'utilisent dans les deux, etc. Enfin les locutions, les mots étrangers, etc. ne sont pas classables dans l'un ou l'autre des deux groupes.

Le tableau 7 présente le poids respectif des groupes nominaux et verbaux chez $\mathrm{S}$. Harper et dans quelques autres corpus de référence (en négligeant les mots étrangers et les locutions) (Labbé et Monière, 2008a et 2008b). Nicolas Sarkozy est, pour l'instant, le seul autre homme politique pour lequel on ait deux corpus : les discours de campagne (N. Sarkozy candidat) et les discours tenus lors des premiers mois de sa présidence (dernière colonne). 
POIDS DES GROUPES NOMINAL ET VERBAL DANS DIVERS CORPUS (EN \%)

\begin{tabular}{|l|c|c|c|c|c|c|}
\hline & $\begin{array}{c}\text { S. Harper } \\
\text { premier } \\
\text { ministre }\end{array}$ & $\begin{array}{c}\text { S. Harper } \\
\text { candidat }\end{array}$ & $\begin{array}{c}\text { Français } \\
\text { écrit }\end{array}$ & $\begin{array}{c}\text { Premiers } \\
\text { ministres } \\
\text { québécois }\end{array}$ & $\begin{array}{c}\text { N. Sarkozy } \\
\text { candidat }\end{array}$ & $\begin{array}{c}\text { N. Sarkozy } \\
\text { président }\end{array}$ \\
\hline Groupe nominal & 73,2 & 71,5 & 63,1 & 64,5 & 59,7 & 62,0 \\
\hline Groupe verbal & 26,8 & 28,5 & 36,9 & 35,5 & 40,3 & 38,0 \\
\hline
\end{tabular}

La troisième colonne indique qu'en moyenne, dans les textes écrits en français, le groupe verbal couvre 37 pour cent de la surface des textes contre 63 pour cent pour le groupe nominal (en négligeant les locutions et mots étrangers). Chez les premiers ministres québécois, les moyennes sont très proches (soit, respectivement, 35,5\% et $64,5 \%$ ). Par rapport à ces moyennes, S. Harper - comme premier ministre aussi bien que comme candidat - présente une forte propension à sur-employer le groupe nominal et à fuir le verbe et ses satellites. Cette propension était encore un peu plus marquée lorsqu'il était premier ministre que lors de la campagne électorale. En fait, l'entrée en campagne électorale ne fait que rapprocher légèrement $S$. Harper de l'usage habituel, sans toutefois corriger sa forte préférence pour le substantif. N. Sarkozy semble être dans la situation inverse, montrant une préférence marquée pour le groupe verbal. Cependant, chez lui aussi, l'emploi du verbe est un peu moins fort quand il est au pouvoir que lorsqu'il mène campagne.

Comment interpréter la nette préférence de S. Harper pour le groupe nominal et sa fuite devant le verbe?

En l'état actuel des recherches, plusieurs interprétations complémentaires sont possibles.

Premièrement, dans son analyse du vocabulaire des présidents de la République française depuis 1958, D. Mayaffre propose l'hypothèse selon laquelle une prépondérance relative du substantif marquerait un discours orienté vers les «finalités de la politique» (les notions, les concepts, les idées) et une augmentation de la densité des verbes déplacerait le centre de gravité du discours vers «les moyens de la politique» (Mayaffre, 2004 : 35-47). Si l'on applique cette hypothèse au discours de S. Harper, on devrait en conclure que la situation confuse des années 2006-2008 l'a conduit à donner 
plus de place aux idées qu'à l'action (Monière, Labbé et Labbé, 2005). Cela paraît logique, et cela cadre assez bien avec la position prudente et attentiste adoptée par le premier ministre, du moins en politique intérieure.

Deuxièmement, pour la stylistique traditionnelle, la construction nominale «présente le fait sans date, sans mode, peut-être sans aspect, sans le rattacher nécessairement à un sujet (donc à une cause), à un objet (donc à un but)» (Cressot, 1963: 154). La linguistique moderne nuance cette proposition de la manière suivante. Le verbe (ou ses équivalents) a une double fonction : la «fonction cohésive» qui organise «en une structure complète les éléments de l'énoncé» et la fonction assertive qui «dote l'énoncé d'un prédicat de réalité», car l'élément verbal implique une référence à un ordre qui n'est plus simplement celui du discours, mais celui de la réalité (Benveniste, 1950). Si l'on adopte ces hypothèses, la préférence pour le groupe nominal permettrait à S. Harper d'effacer de son discours (au moins partiellement) les questions pour lesquelles il n'avait pas la réponse ou qui semblaient hors de la portée de son gouvernement minoritaire.

Troisièmement, dès 1950, le statisticien P. Guiraud avait signalé que le nombre des substantifs et celui des verbes variaient en sens inverse et que le substantif dominait dans la prose abstraite.

Enfin, il a été montré comment, chez le même auteur, le passage de l'oral à l'écrit se traduit par une diminution très notable du poids du groupe verbal et par une augmentation parallèle du groupe nominal (Labbé, 2002). Autrement dit, l'expression spontanée privilégie le verbe, les pronoms, les adverbes. Le passage à l'écrit amène à remplacer un certain nombre de ces verbes par des substantifs, certains adverbes par des adjectifs, à réduire l'emploi du démonstratif, et ainsi de suite. On peut donc penser que l'effort d'élaboration qu'implique l'écrit s'accompagne d'un mouvement d'abstraction audelà de la perception ou de la visée immédiate de l'auteur et que, plus les contraintes formelles pesant sur la communication sont fortes, plus le groupe nominal s'impose au détriment du groupe verbal. Cela expliquerait, en partie, la diminution du groupe verbal observée chez N. Sarkozy quand il est passé de la campagne électorale - propice à une expression plus spontanée - à l'Élysée où les discours sont peut-être un peu plus prudents et mieux élaborés.

Enfin, chez S. Harper, on note la forte densité des participes présents et des adjectifs tirés du participe passé qui sont tous deux corrélés avec un usage important de l'adjectif. 
M. Cressot a signalé les caractéristiques particulières du participe présent, qui est la forme verbale la plus proche de l'adjectif: «Cette forme a pris au $\mathrm{XIX}^{\mathrm{e}}$ siècle un développement considérable, surtout à partir de Flaubert. Les écrivains qui attribuent aux choses une vie et une volonté secrètes, ont compris l'utilité de l'adjectif verbal pour leur expression dynamique du monde, et la possibilité d'atténuer, grâce à lui, la note trop éclatante des adjectifs en -eur et en -teur » (Cressot, $1963: 150)$

Par rapport aux autres corpus de référence cités, S. Harper présente une nette préférence pour l'adjectif. Selon M. Cressot, «la caractérisation n'existe pas nécessairement dans le mot, mais dans une intention de l'esprit qui classe tel détail dans des catégories de valeurs morales ou esthétiques ou simplement descriptives» (Cressot, 1963 : 150). L'adjectif a pour fonction d'indiquer cette intention de l'esprit en ajoutant une caractérisation au substantif (ou au pronom) dont il est l'épithète ou l'attribut. Cette caractérisation peut être à visée essentiellement descriptive. Mais la plupart du temps, l'adjectif ajoute un jugement à la qualité. Le sur-emploi des adjectifs est révélateur de la spécificité des discours électoraux et atteste de leur fonction promotionnelle. Comme cela peut s'observer aussi dans le domaine de la publicité commerciale, dans une logique de compétition électorale, il s'agit avant tout de mettre en valeur des offres de politiques. C'est par l'adjectif qu'on peut le mieux valoriser ses propositions, mais aussi disqualifier celles de ses adversaires.

Enfin, les principaux indicateurs du style viennent compléter ce portrait.

\section{Les indicateurs de style}

Lorsqu'il s'adresse à un auditoire, l'homme politique choisit un certain style de communication. Quatre indicateurs statistiques permettent de caractériser ce choix. Deux portent sur le vocabulaire (diversité et spécialisation) et deux sur la phrase (longueur et complexité relative). Les résultats sont présentés dans le tableau 8. 
TABLEAU 8

LE STYLE DE STEPHEN HARPER AU GOUVERNEMENT ET EN CAMPAGNE ELECTORALE

\begin{tabular}{|l|c|c|}
\hline Indice & Harper premier ministre & Harper candidat \\
\hline Diversité du vocabulaire (pour 10 000 mots) & 1702 & 1420 \\
\hline Spécialisation du vocabulaire (\%) & 26 & 16 \\
\hline Longueur moyenne des phrases (mots) & 25,8 & 21,8 \\
\hline Ponctuation interne (par phrase) & 1,31 & 1,13 \\
\hline
\end{tabular}

Dans sa relation avec l'auditoire et avec l'objet qu'il traite, un orateur peut choisir la diversité de l'expression - il mobilisera alors un vocabulaire étendu au risque de brouiller son message - ou au contraire la simplicité, sans reculer devant la répétition. L'indice de diversité du vocabulaire estime cette première caractéristique. Il mesure le nombre de vocables différents contenus en moyenne dans toute tranche de 10000 mots contigus. D'après les recherches en cours sur les divers corpus déjà signalés, une valeur supérieure à 1500 (vocables différents pour 10000 mots) ne se rencontre qu'à l'écrit. Plus on s'élève au-dessus de ce seuil, plus l'expression est recherchée. Au-dessus de 1850 , le texte peut donner un sentiment de recherche, voire de préciosité. En dessous de 1500 , la forme est orale; les répétitions deviennent sensibles à l'auditeur attentif; la forme peut sembler relâchée.

Un orateur peut aussi choisir d'employer un vocabulaire spécifique pour chaque thème qu'il traite ou, à l'inverse, aborder différents thèmes en utilisant le même vocabulaire. L'indice de spécialisation mesure cette seconde caractéristique en estimant le poids relatif du vocabulaire spécialisé dans le vocabulaire total. En dessus de 20 pour cent - c'est-à-dire qu'un mot sur cinq est tiré d'un lexique technique employé pour traiter un thème et un seul - la spécialisation est forte, ce qui peut donner un sentiment de sérieux, voire de «technicité», et risque d'augmenter la difficulté de la communication. En dessous de 10 pour cent, l'orateur utilise un registre unique, facilitant la tâche du destinataire du message, mais risquant de donner le sentiment qu'il se réfugie dans les généralités ou qu'il connaît mal certains dossiers.

Le discours électoral - parce qu'il a des visées plus immédiatement persuasives que le discours de gouvernement - a tendance à privilégier un style plus répétitif et un 
vocabulaire moins spécialisé. S. Harper n'échappe pas à cette loi : ses discours prononcés à titre de premier ministre étaient plus élaborés, leur vocabulaire était plus divers et plus spécialisé; par contre il a choisi une forme plus simple, plus répétitive et un vocabulaire beaucoup plus général pour s'adresser aux électeurs.

L'étude de la phrase du général de Gaulle, menée par J.-M. Cotteret et R. Moreau (1969 : 42-50), a montré que la longueur des phrases est aussi un indice du style. M. Cressot écrit à ce propos : «La phrase longue répond à une vision totale et complexe et à une volonté de rendu synthétique» (1963 : 207). Elle est l'outil de la démonstration. La phrase courte présente une vision fragmentaire et nette. Elle est plus incisive et appropriée à l'argumentation et à la polémique. Naturellement, un même orateur utilise généralement ces deux types de phrases. Les données du tableau 8 montrent que la phrase du discours électoral est plus courte et moins complexe que celle du discours gouvernemental. Comme l'atteste la simplification de la ponctuation, la phrase électorale est allégée; le souci de l'intelligibilité l'emporte sur le rendu de la complexité des problèmes. 


\section{Conclusions}

Cette étude de cas permet de valider l'hypothèse d'une spécificité du discours électoral puisque le discours électoral du chef conservateur aux élections de 2008 se différencie significativement de ceux qu'il a prononcés à titre de chef de gouvernement. Le discours électoral est plus ancré dans les problèmes du présent et contient plus de références à la communauté, comme le montre notamment le sur-emploi du substantif identitaire «Canadien». C'est aussi un discours qui valorise le sens collectif; le locuteur privilégie l'emploi du «nous» plutôt que de se présenter comme seul responsable des choix collectifs comme il a plus tendance à le faire une fois élu. Comparativement aux discours gouvernementaux, le discours électoral est aussi moins abstrait et plus orienté vers l'action, comme l'indique la prédominance du groupe verbal sur le groupe nominal, de même que l'usage intensif du futur et du verbe «aller». La forte présence de la construction négative, de même que la désignation des adversaires (noms propres), soulignent le caractère polémique du discours électoral. Enfin la fonction promotionnelle du discours électoral a été mise en évidence, notamment par le suremploi des adjectifs.

Les différences de comportements lexicaux observées dans les discours électoraux de S. Harper procèdent-elles d'une idiosyncrasie ou manifestent-elles des caractéristiques propres au discours électoral? D'autres études de cas - comme celle qui est en cours sur N. Sarkozy - seront nécessaires pour généraliser les conclusions qui viennent d'être présentées. Il faudra, entre autres, tenir compte de la variable institutionnelle, qui peut influencer les stratégies discursives, en comparant des discours politiques produits dans le cadre de régimes parlementaires et de régimes présidentiels.

Le cadre restreint de cet article ne permettait pas d'exposer toutes les dimensions de cette comparaison lexicométrique entre les discours gouvernementaux et les discours électoraux. Mais aussi restreinte soit-elle, cette étude débouche sur quelques paradoxes et constats intéressants. Par exemple, c'est quand il entre en campagne électorale pour se faire élire que S. Harper s'efface de son propre discours. En revanche, lorsqu'il parle à titre de premier ministre - qui devrait incarner une majorité et un pays - il centre plus ses propos sur sa propre personne. Autre paradoxe : le discours électoral semble plus tourné vers le mouvement et l'action que le discours de gouvernement. Pourtant en campagne, la seule action du leader se résume à battre les estrades et à parler. En 
revanche, une fois à la tête du gouvernement, le leader semble se dérober et tenir un discours plus statique et plus abstrait.

Cette analyse suggère que la lexicométrie peut éclairer plusieurs questions posées à la science politique. Elle permet de comprendre ce que disent les leaders politiques et de décoder leurs stratégies de communication. Évidemment, le but d'une campagne électorale est de persuader le maximum d'électeurs : les mots sont choisis pour obtenir des votes. Mais, malgré ce qu'affirment les conseillers en communication, il est difficile d'établir une relation entre le contenu des discours des candidats et leur réussite ou leur échec. Les expériences, consistant à tester les effets des discours auprès de petits groupes, ne reproduisent pas la dynamique d'une campagne électorale. Pour ce faire, il faudrait mettre en relation les discours réellement prononcés et l'évolution de l'opinion publique. On pourrait vérifier si les sondages influencent la production des discours politiques en mettant en rapport ces sondages avec les ruptures thématiques dans le discours des candidats. On pourrait aussi relier les intentions de vote, exprimées dans les sondages, avec le contenu des discours prononcés. Cependant, il faudrait tenir compte du décalage inhérent entre l'émission du message, sa transmission - par les leaders et les relais d'opinion - et son éventuelle absorption par les électeurs. La lexicométrie produit les données nécessaires à la première étape de cette vérification en établissant le contenu de ce qui est émis à un moment donné, mais aussi, comme nous venons de le montrer, les thématiques et les stratégies de communication choisies par l'émetteur. Il reste donc à mesurer la relation entre ces données et l'évolution de l'opinion publique pour pouvoir saisir les effets des discours.

\section{Références}

Achard Pierre et Al. 1995. Dire non en politique. Mots 45, décembre.

Benoît Robert et Al. 1985. Le nous politique. Mots 10, mars.

Benveniste, Émile. 1950. «La phrase nominale». Reproduit dans Problèmes de linguistique générale. Tome I. Paris ; Gallimard, 1966, 151-167.

Benveniste, Émile. 1956. «La nature des pronoms». Reproduit dans Problèmes de linguistique générale. Tome I. Paris : Gallimard, 1966, 251-265. 
Benveniste, Émile. 1965. «Structure des relations d'auxiliarité». Reproduit dans Problèmes de linguistique générale. Tome II. Paris : Gallimard, 1970, 177-193.

Cotteret, Jean-Marie et René Moreau. 1969. Le vocabulaire du Général de Gaulle. Paris: Presses de la FNSP.

Coullomb-Gully Marlène (dir). 2009. 2007. Débats pour l'Elysée. Mots 89, mars.

Cressot, Marcel. 1963. Le style et ses techniques. Paris : PUF (1 ${ }^{\text {re }}$ édition en 1947).

Ducrot, Oswald. 1972. Dire et ne pas dire. Paris : Hermann.

Guiraud, Pierre. 1950. Les caractères statistiques du vocabulaire. Paris : PUF.

Kerbrat-Orecchioni, Catherine. 1981. L'énonciation de la subjectivité dans le langage. Paris : A. Colin.

Labbé, Cyril et Dominique Labbé. 1994. Que mesure la spécificité du vocabulaire? Grenoble, CERAT, décembre. Reproduit dans Lexicometrica 3, 2001.

Labbé, Cyril, Dominique Labbé et Denis Monière. 2008. «Les styles discursifs des premiers ministres québécois de Jean Lesage à Jean Charest». Revue canadienne de science politique 41.1 : 43-69.

Labbé, Dominique. 1990a. Le vocabulaire de F. Mitterrand. Paris : Presses de la FNSP.

Labbé, Dominique. 1990b. Normes de saisie et de dépouillement des textes politiques. Grenoble : Cahiers du CERAT.

Labbé, Dominique. 2002. «Le général de Gaulle en campagne ». Communication aux III $^{\mathrm{e}}$ Journées de l'ERLA. Reproduit dans David Banks (ed.) Aspects linguistiques $d u$ texte de propagande. Paris : L'Harmattan, 2005, 213-233.

Labbé, Dominique et Denis Monière. 2003. Le vocabulaire gouvernemental. Canada, Québec, France (1945-2000). Paris : Champion.

Labbé, Dominique et Denis Monière. 2008a. Les mots qui nous gouvernent. Montréal : Monière-Wollank.

Labbé, Dominique et Denis Monière. 2008b. «Des mots pour des voix : 132 discours pour devenir président de la République française». Revue Française de Science Politique 58-3, juin, 433-455.

Lebart, Ludovic et André Salem. 1994. Statistique textuelle. Paris : Dunod.

Mayaffre, Damon. 2004. Paroles de président. Jacques Chirac (1995-2003) et le discours présidentiel sous la $V^{e}$ République. Paris : Honoré Champion. 
Monière, Denis. 1988. Le discours électoral : les politiciens sont-ils fiables? Montréal : Québec-Amérique.

Monière, Denis. 2008. «La guerre des mots : les stratégies de communication des partis aux élections fédérales de 2008». Options Politiques, novembre, 33-37.

Monière, Denis, Labbé Cyril et Dominique Labbé. 2005. «Les particularités d'un discours politique : les gouvernements minoritaires de Pierre Trudeau et de Paul Martin au Canada». Corpus 4 : 79-104.

Monière, Denis et Dominique Labbé. 2008. «Je est un autre?» Dans Serge Heiden et Bénédicte Pincemin (eds). $9^{e}$ Journées internationales d'analyse statistique des données textuelles (Lyon, 12-14 mars 2008). Lyon : Presses universitaires de Lyon, volume 2, 647-656.

Muller, Charles. 1977. Principes et méthodes de statistique lexicale. Paris : Hachette.

Muller, Pierre. 1995. «Jaurès et Guesde. Quand une méthode en nie une autre». Mots 45, décembre, 10-22. 